\title{
PECS II block is associated with lower incidence of chronic pain after breast surgery
}

\author{
Alessandro De Cassai ${ }^{1}$, Claudio Bonanno ${ }^{1}$, Ludovica Sandei ${ }^{1}$, Francesco Finozzi ${ }^{1}$, Michele Carron ${ }^{1}$, and \\ Alberto Marchet ${ }^{2}$
}

'Department of Medicine - DIMED, Section of Anesthesiology and Intensive Care, University of Padova, Padova, Italy

${ }^{2}$ Day Surgery Multidisciplinare, Surgical Department, Azienda Ospedaliera Padova, Padova, Italy

Received May 10, 2019

Revised July 28, 2019

Accepted July 29, 2019

\section{Correspondence}

Alessandro De Cassai

Department of Medicine - DIMED, Section

of Anesthesiology and Intensive Care,

University of Padova, Via Giustiniani 1,

Padova 35127, Italy

Tel: +39-0498213090

Fax: +39-0498213091

E-mail: alessandro.decassai@gmail.com

\section{Key Words}

Analgesics, Opioid

Anesthesia, Local

Breast Neoplasm

Chronic Pain

Clinical Study

Humans

Nerve Block

Pain, Postoperative
Background: Breast cancer is complicated by a high incidence of chronic postoperative pain (25\%-60\%). Regional anesthesia might play an important role in lowering the incidence of chronic pain; however it is not known if the pectoral nerve block (PECS block), which is commonly used for breast surgery, is able to prevent this complication. Our main objective was therefore to detect any association between the PECS block and chronic pain at 3, 6, 9, and 12 months in patients undergoing breast surgery.

Methods: We conducted a prospective, monocentric, observational study. We enrolled 140 consecutive patients undergoing breast surgery and divided them in patients receiving a PECS block and general anesthesia (PECS group) and patients receiving only general anesthesia (GA group). Then we considered both intraoperative variables (intravenous opioids administration), postoperative data (pain suffered by the patients during the first 24 postoperative hours and the need for additional analgesic administration) and development and persistence of chronic pain (at 3, 6, 9, and $12 \mathrm{mo}$ ).

Results: The PECS group had a lower incidence of chronic pain at 3 months $(14.9 \%$ vs. $31.8 \%, P=0.039$ ), needed less intraoperative opioids (fentanyl $1.61 \mu \mathrm{g} / \mathrm{kg} / \mathrm{hr}$ vs. $3.3 \mu \mathrm{g} / \mathrm{kg} / \mathrm{hr}, P<0.001)$ and had less postoperative pain ( $3 \mathrm{vs} .4, P=0.017$ ). Conclusions: The PECS block might play an important role in lowering incidence of chronic pain, but further studies are needed.

\section{INTRODUCTION}

Breast cancer is the most frequent cancer in females, with $2,088,849$ estimated new cases and 626,679 deaths in 2018, worldwide [1]. Despite the relatively high survival rate, patients have to face complications linked to both cancer and cancer-therapy. The main complications are persistent pain after breast cancer treatment (PPBCT), breast cancer related lymphedema, and limitation of arm and shoulder movement. PPBCT is a relatively common post-surgical complication (25\%-60\%) [2] that causes a deterioration of quality of life [3] and increase in health expenditure [4].

The pectoral nerve block (PECS) II block is an interfascial block proposed by Blanco et al. [5] that consists in 2 injections of local anesthetic: the first one between the pectoralis minor muscle (Pmm) and pectoralis major muscle (PMM), and the second one between PMM and serratus muscle (SM). This block can provide good anesthesia for the breast and axilla region; however, it is usually unable to cover the parasternal branches of the intercostal nerves. (c) This is an open-access article distributed under the terms of the Creative Commons Attribution Non-Commercial License (http://creativecommons.org/licenses/by-nc/4.0/), which permits unrestricted non-commercial use, distribution, and reproduction in any medium, provided the original work is properly cited.

(C) The Korean Pain Society, 2019
Author contributions: Alessandro De Cassai: Study conception; Claudio Bonanno: Study conception; Ludovica Sandei: Writing/manuscript preparation; Francesco Finozzi: Investigation; Michele Carron: Supervision; Alberto Marchet: Writing/manuscript preparation. 
The PECS II block has been proven to lower opioids consumption and reduce pain scores in breast surgery [6], but it is still unknown if it could have any role in lowering the incidence of PPBCT.

Our main objective was to detect the efficacy of the PECS block in reducing chronic pain at 3, 6, 9, and 12 months in patients undergoing breast surgery (mastectomy or quadrantectomy). Our secondary objective was to evaluate the role of the PECS block in intraoperative opioid consumption and its postoperative analgesic effect evaluated by both the mean and maximum pain experienced, expressed using the numerical rating scale (NRS) in the first 24 hours, and by the requirement of rescue analgesic therapy.

\section{MATERIALS AND METHODS}

\section{Design}

We conducted a prospective, monocentric, observational study. The study protocol was approved by the Institutional Review Board of University Hospital of Padova (IRB No: 4355/AO/17) in April 2017 and registered on Clinicaltrials.gov (NCT03448913). The study was done in accordance with the 1964 Helsinki declaration and its later amendments or comparable ethical standards.

\section{Subjects and setting}

Informed consent was obtained from all individual participants scheduled for mastectomy or lateral quadrantectomy aged 18 or older. Patients undergoing surgery on both breasts at the same time and patients undergoing medial quadrantectomy were excluded from the study.

\section{Anesthesia}

All patients were given a standardized general anesthetic as per local protocol. Anesthesia was induced with propofol 2 $\mathrm{mg} / \mathrm{kg}$ and fentanyl $2 \mu \mathrm{g} / \mathrm{kg}$, after which a laryngeal mask of appropriate size was utilized, and the patients' lungs were ventilated with a 40/60 oxygen/air mixture using a pressure-regulated volume-control mode (FLOW-i anesthesia machine; MAQUET, Rastatt, Germany). The expiratory tidal volume was maintained at $8 \mathrm{~mL} / \mathrm{kg}$ and $5 \mathrm{cmH}_{2} \mathrm{O}$ PEEP, adjusting the respiratory rate to keep the end tidal carbon dioxide pressure $\left(\mathrm{etCO}_{2}\right)$ at 35 to $40 \mathrm{mmHg}$. Anesthesia was maintained with propofol, fentanyl, and remifentanil to ensure a bispectral index value between 40 and 60 .

All surgical operations were performed by the same surgeon and by 9 different anesthesiologists. Three anes- thesiologists out of 9 were confident with PECS block and used it in their daily practice, 6 anesthesiologists were not confident with the technique and did not execute it in their normal practice.

\section{Block procedure}

The PECS II block was executed in a standardized way as per local protocol. After disinfection, the block was executed as described by Blanco et al. [5] using $30 \mathrm{~mL}$ of levobupivacaine $0.25 \%$ ( $10 \mathrm{~mL}$ between the Pmm and PMM, and $20 \mathrm{~mL}$ between the PMM and SM). The blocks were performed on the PECS group at least 20 minutes before skin incision.

\section{Variables}

We assessed anagraphic data for all the enrolled patients, such as age, weight $(\mathrm{kg})$, height $(\mathrm{cm})$, body mass index $(\mathrm{kg} /$ $\mathrm{cm}^{2}$ ), and other comorbidities. We then collected intraoperative data such as the use of the PECS block and, eventually, the local anesthetic choice, concentration and volume $(\mathrm{mL})$ used, the consumption of intravenous fentanyl $(\mu \mathrm{g} /$ $\mathrm{kg} / \mathrm{hr}$ ), and the duration of the intervention (min). Postoperatively, participants were evaluated using the NRS to record the highest and the average pain experienced during the first 24 postoperative hours, as well as requests for rescue analgesics during the first 24 hours and the eventual development and persistence of chronic pain. Chronic pain was defined as a non-pre-existing pain referred to the breast, axilla, or arm that develops after the surgical procedure, persisting at least 3 months after the intervention, and excluding other causes of pain [7].

The "chronic pain follow-up" has been executed by a telephonic questionnaire at $3,6,9$, and 12 months, using a validated questionnaire [8] with some minor modifications. To determine the prevalence of chronic pain, yes or no questions were used. Regarding pain the women were asked to address 4 specific regions: a) the area of the breast (defined as either the affected breast or the area from which the breast was removed), b) the axilla, c) the arm, and d) the side of the body, rating pain severity and frequency in each region. To estimate the severity of pain, a NRS score from 0 to 10 was used in which 0 indicated no pain and 10 indicated the worst imaginable pain. Pain severity was defined as the highest pain score of the 4 regional pain scores. Patients were excluded from the followup if they did not develop any chronic pain (a) or when it been resolved (b) meaning an absence of referred pain for 30 days or more before follow-up.

To calculate the required sample size, we considered the $47 \%$ chronic pain incidence after breast surgery [8], aiming 
to show a 50\% reduction of PPBCT incidence in the PECS group, compared with the receiving only general anesthesia (GA) group. We used in the calculation a 1:2 enrollment ratio factor considering that only 3 anesthesiologists out of 9 were confident with the PECS block. A group size of 126 patients was calculated to detect the planned PPBCT reduction with a power of $80 \%$ and a significance level of 0.05 . We expected a $10 \%$ patient drop off rate during followup, and we therefore decided to enroll 140 patients in the study. The TREND (transparent reporting of evaluations with nonrandomized designs) statement [9] was followed while preparing this manuscript.

\section{Statistical analysis}

The normality of the distribution of quantitative characteristics was analyzed using the Shapiro-Wilk test. Preoperative and postoperative variables were compared using the 2-tail Student's $t$-test if the variable was normally distributed, or the Mann-Whitney $U$-test if it was non-normally distributed. Continuous variables are presented as mean \pm standard deviation. For non-normally distributed variables, the values of median, first, and third interquartiles are reported. Variables presented as percentages were compared between the 2 groups using the chi-square test or the Fisher exact test when appropriate.

All statistical analyses were conducted using $R$ version 3.4.0 (2017-04-21). $P$ values $<0.05$ were considered statistically significant.

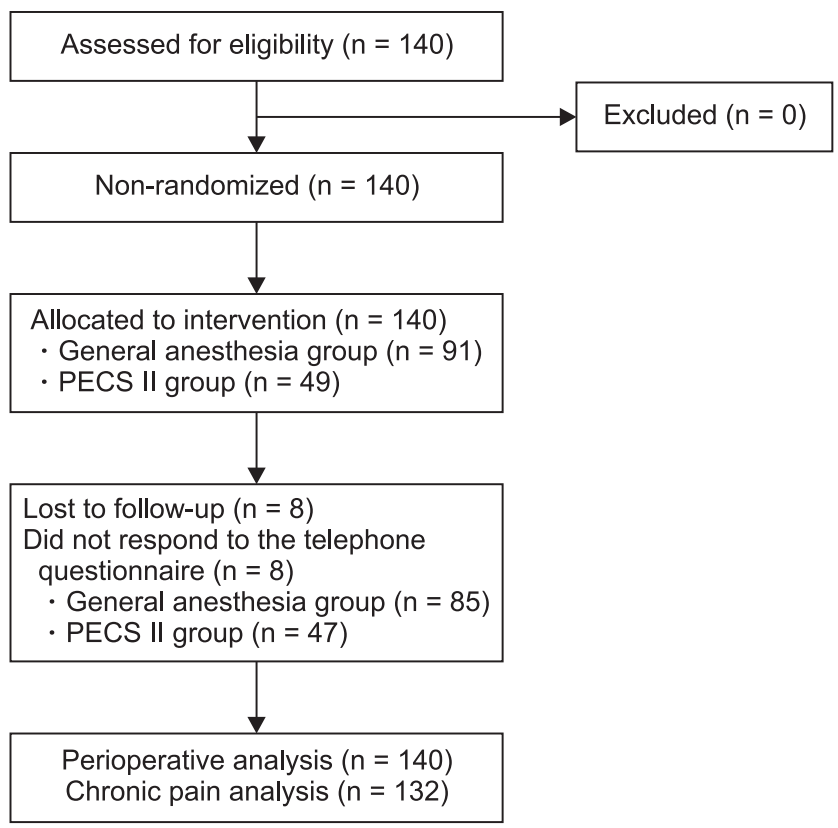

Fig. 1. Study flowchart. PECS: pectoral nerve block.

\section{RESULTS}

One hundred and forty patients undergoing breast surgery (mastectomy or quadrantectomy) were enrolled from February 1, 2018 until July 15, 2018 (Fig. 1). One hundred thirty-seven patients were female $(97.9 \%)$ and 3 patients were male (2.1\%). Fifty-seven patients underwent mastectomy (40.7\%), while 83 underwent quadrantectomy (59.3\%). Forty-nine patients (35.0\%) received a PECS II block as a regional anesthesia technique (from now on "the PECS group") while 91 patients (65.0\%) received no regional anesthesia (from now on "the GA group"). Demographic characteristics and chronic pain predisposing factors are reported in Table 1.

Intraoperative opioid consumption of both fentanyl and remifentanil was lower in the PECS group. In the surgical ward, as a hospital protocol, all patients received acetaminophen $1 \mathrm{~g} / 8 \mathrm{hr}$ and further analgesics if requested by the patient. At 24 postoperative hours, the NRS score was lower in the PECS group, both in worst pain experienced and in average pain. During the first 24 hours after the surgery the GA group requested rescue analgesics more frequently than the PECS group. Group and subgroups analysis for perioperative data are reported in Table 2.

Five patients (one from the PECS group and four from the GA group) never responded to the telephone questionnaire and for this reason they were excluded in the followup. Twenty-seven patients in the GA group and 7 patients in the PECS group developed chronic pain at the 3 months follow-up $(P=0.039)$. Pain was located in the breast $(85.71 \%$ PECS group, $62.96 \%$ GA group), arm (14.29\% PECS group, $22.22 \%$ GA group), and shoulder (0\% PECS group, $14.82 \%$ GA group). The pain was defined as tingling (71.43\% PECS group, 51.85\% GA group), burning (28.57\% PECS group, $25.93 \%$ GA group), shooting (0\% PECS group, $7.41 \%$ GA group), and stinging (0\% PECS group, 14.81\% GA group).

Table 1. Demographic and Chronic Pain Predisposing Factors Data

\begin{tabular}{lccl}
\hline \multicolumn{1}{c}{ Variable } & GA group & PECS group & $P$ value \\
\hline Age $(\mathrm{yr})$ & $61.3 \pm 11.5$ & $63.8 \pm 12.1$ & 0.243 \\
Height $(\mathrm{cm})$ & $162.7 \pm 6.1$ & $164.7 \pm 6.9$ & 0.104 \\
Weight $(\mathrm{kg})$ & $64(58-75)$ & $71(62-80)$ & $0.02^{*}$ \\
BMI $\left(\mathrm{kg} / \mathrm{m}^{2}\right)$ & $24.5(22.0-28.2)$ & $26.1(23.1-29.8)$ & 0.142 \\
CTH $(\%)$ & 27.5 & 14.3 & 0.883 \\
RTH $(\%)$ & 82.4 & 77.6 & 0.48 \\
CPH $(\%)$ & 27.5 & 30.6 & 0.695 \\
\hline
\end{tabular}

Values are presented as mean \pm standard deviation, median (range), or percent only.

GA group: patients receiving only general anesthesia, PECS group: patients receiving a pectoral nerve block and general anesthesia, BMI: body mass index, CTH: chemotherapy history, RTH: radiotherapy history, $\mathrm{CPH}$ : chronic pain history.

*Asterisk indicates a statistically significant $(P<0.05)$. 
Table 2. Group and Subgroups Analysis for Perioperative Data

\begin{tabular}{|c|c|c|c|}
\hline Variable & GA group & PECS group & $P$ value \\
\hline Mastectomy (\%) & 38.5 & 44.9 & 0.459 \\
\hline \multicolumn{4}{|l|}{ Time (min) } \\
\hline Total & $80(60-90)$ & $70(60-90)$ & 0.08 \\
\hline Mastectomy & $90(90-90)$ & $90(90-90)$ & 0.764 \\
\hline Quadrantectomy & $60(50-70)$ & $60(60-60)$ & 0.825 \\
\hline \multicolumn{4}{|l|}{ Fentanyl ( $\mu \mathrm{g} / \mathrm{kg} / \mathrm{hr})$} \\
\hline Total & $3.3(2.2-4.4)$ & $1.61(1.21-2.31)$ & $<0.001 *$ \\
\hline Mastectomy & $2.8(2.1-5.5)$ & $1.53(1.20-2.2)$ & $<0.001 *$ \\
\hline Quadrantectomy & $3.7(3.0-4.6)$ & $1.68(1.31-2.34)$ & $<0.001 *$ \\
\hline \multicolumn{4}{|l|}{ Remifentanil ( $\mu \mathrm{g} / \mathrm{kg} / \mathrm{min})$} \\
\hline Total & $0.1(0-0.1)$ & $0(0-0)$ & $<0.001 *$ \\
\hline Mastectomy & $0.1(0-0.1)$ & $0(0-0.0)$ & $<0.001 *$ \\
\hline Quadrantectomy & $0(0-0.1)$ & $0(0-0)$ & $<0.001 *$ \\
\hline \multicolumn{4}{|l|}{ Mean NRS } \\
\hline Total & $3(1-4)$ & $1(0-3)$ & $0.005^{*}$ \\
\hline Mastectomy & $4(1-5)$ & $1(0.3-3)$ & $0.02^{*}$ \\
\hline Quadrantectomy & $2(1-3)$ & $1(0-3)$ & 0.06 \\
\hline \multicolumn{4}{|l|}{ Max NRS } \\
\hline Total & $4(2-6)$ & $3(1-4)$ & $0.017 *$ \\
\hline Mastectomy & $5(3-8)$ & $2(0-3)$ & $0.02 *$ \\
\hline Quadrantectomy & $3(1-5)$ & $2(1-4)$ & 0.12 \\
\hline \multicolumn{4}{|l|}{ Analg. Req. } \\
\hline Total (\%) & 37.4 & 14.3 & $0.006^{*}$ \\
\hline Mastectomy (\%) & 48.6 & 27.3 & 0.166 \\
\hline Quadrantectomy (\%) & 30.4 & 3.7 & 0.005 \\
\hline
\end{tabular}

Values are presented as percent only or median (range).

GA group: patients receiving only general anesthesia, PECS group: patients receiving a pectoral nerve block and general anesthesia, NRS: numeric rating scale, Analg. Req.: request for analgesic during first postoperative day.

*Asterisk indicates a statistically significant $(P<0.05)$.

Chronic pain lasted 6 months in 3 patients from the PECS group and in 14 patients from the GA group; at 9 months pain persisted in one patient from the PECS group and in five patients from the GA group and was reported at the one year follow-up in one patient from the PECS group and in four patients from the GA group. Subgroup analysis is reported in Table 3. There was no difference in pain intensity at any time between the 2 groups $(P>0.05)$ or in any subgroup.

\section{DISCUSSION}

The main finding of our study is an association between a lower incidence of chronic pain and PECS II at 3 months after breast surgery (31.8\% in the GA group, $14.9 \%$ in the PECS group, $P=0.039$ ). Patients receiving a PECS II block also had a lower incidence of chronic pain at other followup checks, however these results were not statistically relevant at 6,9 , and 12 months after surgery.

Regional anesthesia preventing chronic pain is not in-
Table 3. Postoperative Chronic Pain Incidence at 3, 6, 9, and 12 Months

\begin{tabular}{lccl}
\hline \multicolumn{1}{c}{ Variable } & PECS group & GA group & $P$ value \\
\hline 3 mo & & & \\
Total & 14.9 & 31.8 & $0.039 *$ \\
Quadrantectomy & 15.4 & 30.2 & 0.180 \\
$\quad$ Mastectomy & 14.3 & 34.4 & 0.125 \\
6 mo & & & \\
Total & 6.4 & 16.5 & 0.151 \\
$\quad$ Quadrantectomy & 3.9 & 15.1 & 0.258 \\
$\quad$ Mastectomy & 9.5 & 18.8 & 0.455 \\
9 mo & & & \\
Total & 2.1 & 6.2 & 0.421 \\
Quadrantectomy & 3.9 & 4.1 & 0.707 \\
$\quad$ Mastectomy & 0 & 9.4 & 0.254 \\
12 mo & & & \\
Total & 2.1 & 4.7 & 0.654 \\
Quadrantectomy & 3.9 & 1.9 & 0.552 \\
Mastectomy & 0 & 9.4 & 0.268 \\
\hline
\end{tabular}

Values are presented as percent only.

PECS group: patients receiving a pectoral nerve block and general anesthesia, GA group: patients receiving only general anesthesia.

*Asterisk indicates a statistically significant $(P<0.05)$.

novative for either breast surgery or other types of surgery. In a recent systematic review [10] a paravertebral block reduced chronic pain in patients undergoing breast surgery (odds ratio, $0.61 ; 95 \%$ confidence interval, 0.39-0.97). This result is in accordance with the concept that a patient suffering lesser acute perioperative pain is more unlikely to develop chronic pain symptoms via spinal sensitization [11]. The reduction of chronic pain in breast surgery after a PECS II block is, though, unprecedented.

Continuous stimulation caused by preoperative and acute perioperative pain have been correlated with chronic pain development in randomized controlled trials [10], for this reason an aggressive pain treatment in the perioperative period is of paramount importance [12].

In this frame regional anesthesia appears to be the ideal technique in breast surgery; in fact, regional anesthesia could provide profound and selective analgesia to surgery, avoiding the systemic collateral effects of opioids; moreover, interfascial blocks such as the PECS block technique also permit avoidance of possible risks related to neuraxial and paravertebral techniques. However, evidence supporting this idea is still limited: on one hand, trials showing a benefit are small studies weakened by performance bias, as well as shortcomings in allocation concealment, considerable attrition and incomplete outcome data [10]; on the other hand, some well conducted randomized controlled trials have showed no benefit from preoperative regional anesthesia on chronic pain development [13].

This study also confirms results from previous literature showing that the PECS block is able to reduce intraoperative opioid consumption and perioperative pain in breast 
surgery.

Two studies have evaluated the efficacy of the PECS block in reducing perioperative opioid consumption and pain $[14,15]$ compared to general anesthesia without blocks. Cros et al. [14] concluded that PECS II is superior to general anesthesia alone in providing analgesia in patients undergoing modified radical mastectomy. Bashandy and Abbas [15] concluded that PECS I is superior to general anesthesia alone in providing analgesia in patients undergoing radical mastectomy, but not in patients undergoing quadrantectomy. As for opioid consumption, our results confirm those from previous studies: patients undergoing breast surgery with a PECS II block required less opioids in perioperative periods than patients without a PECS II block.

Cros et al. [14] reported that pain was statistically significantly different, although not clinically significant, for patients undergoing mastectomies or axillary clearance, while Bashandy and Abbas [15] reported lower visual analog scale pain scores if a PECS block was executed.

In our study we found that pain was lower in the PECS group than in the GA group, however we can hardly state that pain difference were clinically relevant using only the NRS score (a difference of 2 points on the NRS); nevertheless, the percentage difference of requests for analgesics between the 2 groups was clinically $(23.07 \%)$ and was statistically significant, thus reinforcing the idea of a real clinical difference in pain between the 2 groups. The reduction of intraoperative opioid consumption in the PECS group confirms that this block is able to provide intraoperative analgesia. However, opioids, and specifically remifentanil, may play a role in acute opioid tolerance and in opioid-induced hyperalgesia by a mechanism that is still not clear [16]; this mechanism could raise some concerns about the role of opioid-induced hyperalgesia in the development of chronic pain in our patients. However, hyperalgesia is thought to be induced when remifentanil is administered at a dose at least $0.25 \mu \mathrm{g} / \mathrm{kg} / \mathrm{min}$ for acute opioid tolerance and $0.2 \mu \mathrm{g} / \mathrm{kg} / \mathrm{min}$ for opioid-induced hyperalgesia for a sustained period of time [16], while in our study patients have received low remifentanil doses for a relatively short duration of the surgical intervention (median dose $0 \mu \mathrm{g} / \mathrm{kg} / \mathrm{min}$ for the PECS group and $0.05 \mu \mathrm{g} / \mathrm{kg} /$ min for the GA group) making a link between remifentanil and chronic pain unlikely.

Our study has some limitations that need to be discussed. The monocentric and observational nature of the study does not allow us to be conclusive about the relationship between PPBCT and the PECS block. In our study we enrolled both mastectomy and quadrantectomy patients, however using 2 types of surgery could be misleading.

The different size of the 2 groups is a limitation of our study because we could not know the exact effect of the different weight on the results. Moreover, not all anesthesiologists performed the block, in fact only 3 anesthesiologists out of 9 were confident with the PECS block and used it in their daily activity, so we recognize this as a limitation of our study. We specifically asked our patients about pain sensations, however we could have also asked about paraesthesia, arm/shoulder swelling, phantom sensations or allodynia.

Moreover, the use of a single analgesic for postoperative pain would be more appropriate to compare the 2 groups.

In conclusion, our study suggests that the PECS II block could be able to prevent chronic pain after breast surgery at 3 months after surgery. However, the specific design of the study and its limitations do not permit to us to be conclusive about this argument. For this reason, well designed randomized controlled studies are necessary to further investigate the relationship between chronic pain and the PECS II block in breast surgery.

\section{CONFLICT OF INTEREST}

No potential conflict of interest relevant to this article was reported.

\section{ORCID}

Alessandro De Cassai, https://orcid.org/0000-0002-9773-1832 Claudio Bonanno, https://orcid.org/0000-0002-8065-4906 Ludovica Sandei, https://orcid.org/0000-0002-3288-1696

Francesco Finozzi, https://orcid.org/0000-0002-3014-5935

Michele Carron, https://orcid.org/0000-0001-5104-4124

Alberto Marchet, https://orcid.org/0000-0002-8912-0505

\section{REFERENCES}

1. International Agency for Research on Cancer (IARC). Estimated number of new cases in 2018, worldwide, both sexes, all ages [Internet]. Lyon: IARC. Available at: http://gco.iarc. fr/today/online-analysis-table.

2. Andersen KG, Kehlet H. Persistent pain after breast cancer treatment: a critical review of risk factors and strategies for prevention. J Pain 2011; 12: 725-46.

3. Peuckmann V, Ekholm O, Rasmussen NK, Groenvold M, Christiansen P, Møller S, et al. Chronic pain and other sequelae in long-term breast cancer survivors: nationwide survey in Denmark. Eur J Pain 2009; 13: 478-85.

4. Blyth FM, March LM, Cousins MJ. Chronic pain-related disability and use of analgesia and health services in a Sydney 
community. Med J Aust 2003; 179: 84-7.

5. Blanco R, Fajardo M, Parras Maldonado T. Ultrasound description of Pecs II (modified Pecs I): a novel approach to breast surgery. Rev Esp Anestesiol Reanim 2012; 59: 470-5.

6. Versyck B, Van Houwe P, van Geffen GJ, Van de Velde M, Slappendel R. A qualitative systematic review of the pectoral nerves block type I and II. Acta Anaesthesiol Belg 2017; 68: 49-62.

7. Treede RD, Rief W, Barke A, Aziz Q, Bennett MI, Benoliel R, et al. A classification of chronic pain for ICD-11. Pain 2015; 156: 1003-7.

8. Gärtner R, Jensen MB, Nielsen J, Ewertz M, Kroman N, Kehlet $\mathrm{H}$. Prevalence of and factors associated with persistent pain following breast cancer surgery. JAMA 2009; 302: 1985-92.

9. Des Jarlais DC, Lyles C, Crepaz N; TREND Group. Improving the reporting quality of nonrandomized evaluations of behavioral and public health interventions: the TREND statement. Am J Public Health 2004; 94: 361-6.

10. Weinstein EJ, Levene JL, Cohen MS, Andreae DA, Chao JY, Johnson $\mathrm{M}$, et al. Local anaesthetics and regional anaesthesia versus conventional analgesia for preventing persistent postoperative pain in adults and children. Cochrane Database Syst Rev 2018; 6: CD007105.

11. Tasmuth T, Kataja M, Blomqvist C, von Smitten K, Kalso E.
Treatment-related factors predisposing to chronic pain in patients with breast cancer--a multivariate approach. Acta Oncol 1997; 36: 625-30.

12. Kraychete DC, Sakata RK, Lannes Lde O, Bandeira ID, Sadatsune EJ. Postoperative persistent chronic pain: what do we know about prevention, risk factors, and treatment. Braz J Anesthesiol 2016; 66: 505-12.

13. Albi-Feldzer A, Mouret-Fourme E E, Hamouda S, Motamed C, Dubois PY, Jouanneau L, et al. A double-blind randomized trial of wound and intercostal space infiltration with ropivacaine during breast cancer surgery: effects on chronic postoperative pain. Anesthesiology 2013; 118: 318-26.

14. Cros J, Sengès P, Kaprelian S, Desroches J, Gagnon C, Labrunie A, et al. Pectoral I block does not improve postoperative analgesia after breast cancer surgery: a randomized, double-blind, dual-centered controlled trial. Reg Anesth Pain Med 2018; 43: 596-604.

15. Bashandy GM, Abbas DN. Pectoral nerves I and II blocks in multimodal analgesia for breast cancer surgery: a randomized clinical trial. Reg Anesth Pain Med 2015; 40: 68-74.

16. Yu EH, Tran DH, Lam SW, Irwin MG. Remifentanil tolerance and hyperalgesia: short-term gain, long-term pain? Anaesthesia 2016; 71: 1347-62. 